Bandt-Pompe symbolization dynamics for time series with tied values: A data-driven approach

Francisco Traversaro, Francisco O. Redelico, Marcelo R. Risk, Alejandro C. Frery, and Osvaldo A. Rosso

Citation: Chaos 28, 075502 (2018); doi: 10.1063/1.5022021

View online: https://doi.org/10.1063/1.5022021

View Table of Contents: http://aip.scitation.org/toc/cha/28/7

Published by the American Institute of Physics

Chaos

An Interdisciplinary Journal of Nonlinear Science

Fast Track Your Research. Submit Today! 


\title{
Bandt-Pompe symbolization dynamics for time series with tied values: A data-driven approach
}

\author{
Francisco Traversaro, ${ }^{1, \text { a) }}$ Francisco O. Redelico, ${ }^{2,3, b)}$ Marcelo R. Risk, ${ }^{4,5, c)}$ \\ Alejandro C. Frery, ${ }^{6, d)}$ and Osvaldo A. Rosso $\left.2,7,8, e\right)$ \\ ${ }^{1}$ Grupo de Investigación en Sistemas de Información, Universidad Nacional de Lanús \& CONICET Lanús, \\ 29 de Septiembre 3901, Buenos Aires B1826GLC, Argentina \\ ${ }^{2}$ Departamento de Informática en Salud, Hospital Italiano de Buenos Aires \& CONICET, \\ Ciudad Autónoma de Buenos Aires C1199ABB, Argentina \\ ${ }^{3}$ Departamento de Ciencia y Tecnología, Universidad Nacional de Quilmes, Roque Sáenz Peña 352, \\ Bernal, Buenos Aires B1876BXD, Argentina \\ ${ }^{4}$ Instituto Tecnólgico de Buenos Aires (ITBA) \& CONICET, Av. Eduardo Madero 399, Ciudad Autónoma de \\ Buenos Aires C1181ACH, Argentina \\ ${ }^{5}$ Departamento de Informática en Salud, Hospital Italiano de Buenos Aires, \\ Ciudad Autónoma de Buenos Aires C1199ABB, Argentina \\ ${ }^{6}$ Laboratório de Computação Científica e Análise Numérica, Universidade Federal de Alagoas, \\ Av. Lourival Melo Mota, s/n, Maceió, Alagoas 57072-970, Brazil \\ ${ }^{7}$ Universidade Federal de Alagoas, Instituto de Física, \\ Av. Lourival Melo Mota, s/n, Maceió, Alagoas 57072-970, Brazil \\ ${ }^{8}$ Complex Systems Group, Facultad de Ingeniería y Ciencias Aplicadas, Universidad de los Andes, \\ Las Condes, Santiago 12455, Chile
}

(Received 10 January 2018; accepted 10 April 2018; published online 11 July 2018)

In 2002, Bandt and Pompe [Phys. Rev. Lett. 88, 174102 (2002)] introduced a successfully symbolic encoding scheme based on the ordinal relation between the amplitude of neighboring values of a given data sequence, from which the permutation entropy can be evaluated. Equalities in the analyzed sequence, for example, repeated equal values, deserve special attention and treatment as was shown recently by Zunino and co-workers [Phys. Lett. A 381, 1883 (2017)]. A significant number of equal values can give rise to false conclusions regarding the underlying temporal structures in practical contexts. In the present contribution, we review the different existing methodologies for treating time series with tied values by classifying them according to their different strategies. In addition, a novel data-driven imputation is presented that proves to outperform the existing methodologies and avoid the false conclusions pointed by Zunino and co-workers. Published by AIP Publishing. https:// doi.org/10.1063/1.5022021

In this work, we propose computational affordable solutions for the problem of tied observations in time series. Such observations may lead to wrong conclusions when using Causal Information Theory descriptors. We compare several strategies and show the ones more apt to solve this problem.

\section{INTRODUCTION}

Physics, as well as other scientific natural disciplines, can be considered an observational science, that is, it tries to infer properties of an unfamiliar system from the analysis of temporal sequences of observations of its behavior, commonly called time series.

Dynamical systems are systems that evolve in time. In practice, one may only be able to measure a scalar time series $\mathcal{X}(t)$ which may be a function of unobserved variables. Then,

\footnotetext{
a) ftraversaro@itba.edu.ar

b) francisco.redelico@gmail.com

c) marcelo.risk@hospitalitaliano.org.ar

d) acfrery@laccan.ufal.br

e) oarosso@gmail.com
}

the natural question is, how much we can learn about the dynamics of the system, i.e., about the variables that characterized it, from $\mathcal{X}(t)$ ? In a more formal way, given a system, be it natural or man-made, and given an observable of such system whose evolution can be tracked in time, how much information is encoded in this observable about the dynamics of the underlying system?

Time series are usually studied either in the time or the frequency domain. ${ }^{1}$ This approach focuses on the time series itself and may not be effective to reveal the underlying dynamics.

A new approach consists in transforming the time series, via a nonparametric transformation, into a sequence of patterns and then making inference over these patterns. With this, the analysis gains robustness and becomes apt to detect relevant causal information related to the unobserved variables that control the system.

Bandt and Pompe (BP) ${ }^{2}$ proposed a very successful technique for dealing with time series under this approach. They computed the Shannon entropy from the histogram of causal patterns and were able to identify chaotic components in sequence of words, among other applications.

Later, Rosso et $a l^{3}$ added an extra dimension to this analysis by considering also the Statistical Complexity 
obtained from the same histogram of causal patterns. The authors have been making contributions to a number of applications. This approach, which employs the ComplexityEntropy plane, has been successfully used in visualization and characterization of different dynamical regimes when the system parameters change, ${ }^{4-11}$ optical chaos, ${ }^{12-16}$ hydrology,,${ }^{17-19}$ geophysics, ${ }^{20-22}$ engineering, ${ }^{23-26}$ biometrics, ${ }^{27}$ characterization of pseudo-random number generators, ${ }^{28,29}$ biomedical signal analysis (Ref. 30 and references therein ${ }^{31-40}$ ), and econophysics (Ref. 30 and references therein ${ }^{41-46}$ ), just to mention a few.

The ordinal pattern methodology introduced by $\mathrm{BP}^{2}$ has provided important new insight into the characterization of experimental and theoretical time series, in particular, in the sensible area of distinction between chaotic-deterministic and stochastic dynamics. ${ }^{3,47-51}$

Amigó and coworkers consider "local" characteristics of the components of the Bandt-Pompe probability distribution function (BP-PDF) ${ }^{50,51}$ showing the following:

- For time series generated by deterministic-chaotic dynamics not all the possible ordinal patterns can be effectively materialized on orbits, calling these kind of patterns "forbidden."

- The emergence of these forbidden patterns represents of a particular property of some elements of the BP-PDF associated with the time series under study.

- The existence of these forbidden ordinal patterns becomes a persistent fact that can be regarded as a "new" dynamical property.

- For a fixed pattern length $D$ (embedding dimension), the number of forbidden patterns of a time series (unobserved patterns) is independent of the series' length $T$, and presents a super-exponential behavior with $D$. It is important to remark that this independence is not shared by other properties of the series, such as proximity and correlation, which die out with time.

Stochastic processes may also display forbidden patterns. ${ }^{47,48,52}$ However, in the case of either uncorrelated (white noise) or correlated stochastic processes (noise with power-law spec$\operatorname{trum} f^{-k}$ with $k>0$, fractional Brownian motion and fractional Gaussian noise), it can be numerically ascertained that no forbidden patterns emerge. In fact, for time series generated by unconstrained stochastic processes (uncorrelated processes), every ordinal pattern has the same probability of appearance,,$^{50-52}$ thus, if the data set is long enough all ordinal patterns will eventually appear. In this case, as the number of time series observations increases, the associated PDF becomes uniform, and the number of observed patterns will depend only on the time series length $T$.

For correlated stochastic processes, the probability of observing a specific individual pattern depends not only on the time series length $T$, but also on the correlation structure. ${ }^{52}$ The existence of a non-observed ordinal pattern does not qualify it as "forbidden," only as "missing," and this could be due to the time series finite length. A similar observation also holds for the case of real data that always possess a stochastic component due to the omnipresence of dynamical noise. ${ }^{53,54}$
Thus, the existence of "missing ordinal patterns" could be either related to stochastic processes (correlated or uncorrelated) or to deterministic noisy processes (always the case for observational time series).

Recently, Rosso and coworkers ${ }^{49}$ showed that even when the presence of forbidden patterns is a characteristic of chaotic dynamics, a minimum pattern-length $D_{\min }$ is needed to detect their presence, in the case of chaotic time series of finite length. In addition, if forbidden ordinal patterns are observed in this case, their number grows exponentially with patternlengths (embedding dimension) $D$, as opposed to the superexponential behavior described by Amigó and coworkers, valid only for the case $D \rightarrow \infty .^{50,51}$

In summary, a minimum pattern-length $D_{\min }$ is needed to detect the presence of forbidden patterns, the hallmark of determinism (chaotic dynamics). This fact has not been previously pointed out in the literature and ignoring it could be the source of erroneous interpretations.

Per contra, in the case of quantifiers evaluated making use of the global characteristics of the permutation BandtPompe PDF, a specific behavior emerges in the case of chaotic dynamics that provides a more "robust" distinction between deterministic and stochastic dynamics. 3 ,47-49

We use the Bandt-Pompe symbolization, which captures temporal causality features. This transformation is based on the relative amplitude of the time series values without any model assumptions. We then quantify such structure using Information Theory descriptors.

Theoretically, sequences of random deviates with uncountable support have zero probability of tied values. In practice, since data are recorded, stored, and transmitted with finite precision, ties may occur.

It is well known since the seminal work of Bandt and Pompe $^{2}$ that the way to handle equal values in the time series data must be treated with care. However, most works have ignored this fact. Recently, Zunino and co-authors ${ }^{55}$ showed that the way ties are handled may have significant consequences when estimating the ordinal patterns probability distribution: their numerical analyses demonstrated that the BP-PDF values are biased as a consequence of the presence of ties in the records. In fact, experimental data digitized with relatively low resolution could have a non-negligible number of ties and, consequently, the BP-PDF estimations may be significantly affected.

Equal values are usually ranked according to their order of appearance. This way of dealing with them introduces non-negligible spurious temporal correlations that can potentially lead to erroneous conclusions about the true underlying dynamic nature. Several authors suggested modifications in the estimation of permutation entropy in face of repeated values. They either do this by giving new rules to sort the data, ${ }^{2,33}$ or by extending the symbolization alphabet. ${ }^{56}$

We propose a new strategy to deal with this issue. Our methodology uses the information of the actual time series to deal with ties. We assume that there is an underlying (unobserved) time series with no ties, and that the data at hand are a corrupted version. With this in mind, our proposal estimates the true patterns from the observed ones using the 
information available from the corrupted data through a suitable probability distribution. It can be seen as a Data-Driven methodology.

The present work is organized as follows: In Sec. II, the ordinal patterns are defined and the different ways to mapping to a symbolic alphabet are reviewed. The permutation Shannon entropy is introduced in Sec. III and the results for mapping with symbolic alphabet of time series with ties are presented in Sec. IV. In Sec. V, the performance of different strategies previously introduced are evaluated in the treatment of decimal expansion of irrational numbers and with time series data from simulated chaotic maps processes recorded with low precision.

\section{ORDINAL PATTERNS AND THE MAPPINGS TO A SYMBOLIC ALPHABET}

Let $\mathcal{X} \equiv\left\{x_{t}\right\}_{t=1}^{T}$ be a realization of a data generator process in the form of a real valued time series of length $T$, and at first, we assume that there is not equal amplitude values in the time series, that is, the probability $P\left(x_{t_{1}}=x_{t_{2}}\right)=0 \forall t_{1} \neq t_{2}$. As was stated by Bandt and Pompe in their seminal paper ${ }^{2}$ : "If the $\left\{x_{t}\right\}_{t=1}^{T}$ attain infinitely many values, it is common to replace them by a symbol sequence $\Pi \equiv\left\{\pi_{j}\right\}$ with finitely many symbols, and calculate source entropy from it." Also, as stressed by Bandt and Pompe in Ref. 2 "the corresponding symbol sequence must come naturally from the $\left\{x_{t}\right\}_{t=1}^{T}$ without former model assumptions."

Let $\mathfrak{A}_{D}$ (with $D \geq 2$ and $D \in \mathbb{Z}$ ) the symmetric group of order $D$ ! form by all possible permutation of order $D$, and the symbol component vector $\pi^{(D)}=\left(\pi_{1}, \pi_{2}, \ldots, \pi_{D}\right)$, so every element in the vector $\pi^{(D)}$ is unique $\left(\pi_{j} \neq \pi_{k} \forall j \neq k\right)$. Consider for the time series $\mathcal{X} \equiv\left\{x_{t}\right\}_{t=1}^{T}$, its time delay embedding representation, with embedding dimension $D \geq 2(D \in \mathbb{Z})$ and time delay $\tau \geq 1(\tau \in \mathbb{Z})$

$$
\mathbf{X}_{t}^{(D, \tau)}=\left(x_{t}, x_{t+\tau}, \ldots, x_{t+(D-1) \tau}\right),
$$

for $t=1,2, \ldots, N$ with $N=T-(D-1) \tau$. In the following and without loss of generality we take $\tau=1$. Then the vector $\mathbf{X}_{t}^{(D)}$ can be mapped to a symbol vector $\boldsymbol{\pi}^{(D)} \in \mathfrak{A}_{D}$. This mapping should be defined in a way that preserves the desired relation between the elements $x_{t} \in \mathbf{X}_{t}^{(D)}$ and all $t \in T$ that shares this pattern has to be mapped to the same $\pi^{(D)}$. In the literature that encompass the permutation Bandt and Pompe symbolization, there are two ways to define the mapping $\mathbf{X}^{(D)} \mapsto \pi^{(D)}$ : (a) ordering the ranks of the $x_{t} \in \mathbf{X}^{(D)}$ in chronological order (Rank Permutation) or, (b) ordering the time indexes according to the ranks of $x_{t} \in \mathbf{X}^{(D)}$ (Chronological Index Permutation).

\section{A. Rank permutation mapping}

For a given but otherwise arbitrary time $t$ and its time delay embedding associate vector of dimension $D, \mathbf{X}_{t}^{(D)}=$ $\left(x_{t}, x_{t+1}, \ldots, x_{t+D-1}\right)$, this is mapped onto the unique vector symbol $\boldsymbol{\pi}^{(D)}=\left(R\left[x_{t}\right], R\left[x_{t+1}\right], \ldots, R\left[x_{t+D-1}\right]\right) \in \mathfrak{A}_{D}$ formed by the rank of its components.
The rank function is defined as:

$$
R\left[x_{t+n}\right]=\sum_{k=0}^{D-1} \mathbb{I}\left(x_{t+k} \leq x_{t+n}\right) \quad \text { for } \quad n=0, \ldots, D-1,
$$

where $\mathbb{I}$ is the indicator function: $\mathbb{I}(\bullet)=1$ if $\bullet$ is true and 0 otherwise. Note that for all the vectors components $x_{t+n} \in$ $\mathbf{X}_{t}^{(D)}$ will be $1 \leq R\left(x_{t+n}\right) \leq D$, and in consequence, the complete alphabet is all the possible permutation of the ranks. We denoted the corresponding ordinal permutation pattern associated with the vector $\pi^{(D)}$ written all its components values in its sequential order and denoted by $\tilde{\pi}^{(D)}$.

For example, let us take the time series with seven values $(T=7)$ given by Bandt and Pompe in their original work ${ }^{2}$ and consider embedding dimension $D=3$ :

$$
\mathcal{X} \equiv\left\{x_{t}\right\}_{t=1}^{T=7} \equiv\{4,7,9,10,6,11,3\}
$$

The five time delay embedding associated vectors and the corresponding patterns obtained by rank permutation mapping are:

$$
\begin{aligned}
& \mathbf{X}_{1}^{(3)}=\left(x_{1}, x_{2}, x_{3}\right)=(4,7,9) \mapsto \tilde{\pi}^{(3)}=123, \\
& \mathbf{X}_{2}^{(3)}=\left(x_{2}, x_{3}, x_{4}\right)=(7,9,10) \mapsto \tilde{\pi}^{(3)}=123, \\
& \mathbf{X}_{3}^{(3)}=\left(x_{3}, x_{4}, x_{5}\right)=(9,10,6) \mapsto \tilde{\pi}^{(3)}=231, \\
& \mathbf{X}_{4}^{(3)}=\left(x_{4}, x_{5}, x_{6}\right)=(10,6,11) \mapsto \tilde{\pi}^{(3)}=213, \\
& \mathbf{X}_{5}^{(3)}=\left(x_{5}, x_{6}, x_{7}\right)=(6,11,3) \mapsto \tilde{\pi}^{(3)}=231 .
\end{aligned}
$$

With this Rank Permutation Mapping, one simply maps each value $x_{t}$ in $\mathbf{X}^{(D)}$ placing its rank $R\left[x_{t}\right] \in\{1,2, \ldots, D\}$ in chronological order in $\boldsymbol{\pi}^{(D)} \in \mathfrak{A}_{D}$. In Fig. 1, an illustrative drawing of this mapping for all alternatives in $D=3$ is presented. It can be seen that the indexes of the vertical axis are fixed, ordered by amplitude (i.e ranks), and they are mapped onto the time axis. The resultant pattern can be obtained reading the labels in the horizontal axis from left to right (in chronological order). This method is used by Refs. 57 and 58, among others.

\section{B. Chronological index permutation mapping}

Again, any $\mathbf{X}_{t}^{(D)}=\left(x_{t}, x_{t+1}, \ldots, x_{t+D-1}\right)$ can be rearranged in increasing order respect to their amplitudes. In order to do the mapping to $\pi^{(D)}=\left(i_{1}, i_{2}, \ldots, i_{D}\right) \in \mathfrak{A}_{D}$, the sequence must comply $x_{t+i_{1}}<x_{t+i_{2}}<\cdots<x_{t+i_{D}}$. Thus, the time indexes are ordered according to their amplitudes. The complete alphabet is again, all the possible permutations of these chronological indexes.

Let us take the previous time series (3) as an example, for $D=3$, the five time delay embedding associated vectors and the corresponding patterns obtained by chronological index mapping are

$$
\begin{aligned}
& \mathbf{X}_{1}^{(3)}=\left(x_{1}, x_{2}, x_{3}\right)=(4,7,9) \mapsto \tilde{\pi}^{(3)}=123, \\
& \mathbf{X}_{2}^{(3)}=\left(x_{2}, x_{3}, x_{4}\right)=(7,9,10) \mapsto \tilde{\pi}^{(3)}=123, \\
& \mathbf{X}_{3}^{(3)}=\left(x_{3}, x_{4}, x_{5}\right)=(9,10,6) \mapsto \tilde{\pi}^{(3)}=312,
\end{aligned}
$$


123

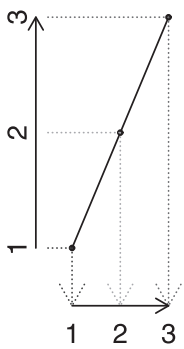

132

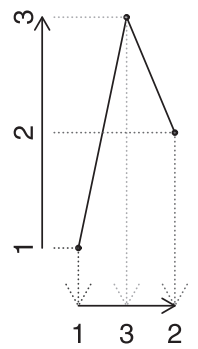

213

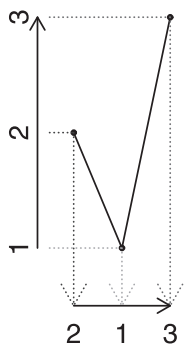

312

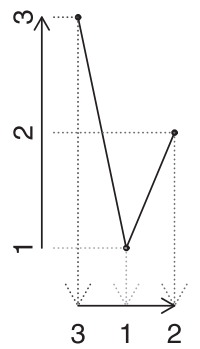

231 321

FIG. 1. Rank permutation mapping: All symbols for $D=3$ are shown. With this Rank Alphabet one simply maps each value $x_{i}$ in the corresponding embedded vector $\mathbf{X}_{t}^{(D)}$ placing its rank in chronological order in $\boldsymbol{\pi}^{(D)}$. It can be seen that the indexes of the vertical axis are fixed, ordered by amplitude (i.e., ranks), and they are mapped onto the time axis. For each vector $\mathbf{X}_{t}^{(3)}=\left(x_{t}, x_{t+1}, x_{t+2}\right)$, the resulting pattern $\tilde{\pi}^{(3)}$ can be obtained reading the labels in the horizontal axis from left to right (in chronological order).

$$
\begin{aligned}
& \mathbf{X}_{4}^{(3)}=\left(x_{4}, x_{5}, x_{6}\right)=(10,6,11) \mapsto \tilde{\pi}^{(3)}=213 \\
& \mathbf{X}_{5}^{(3)}=\left(x_{5}, x_{6}, x_{7}\right)=(6,11,3) \mapsto \tilde{\pi}^{(3)}=312 .
\end{aligned}
$$

We show an illustrative drawing of this mapping for all alternatives in $D=3$ in Fig. 2. The indexes of the time axis are fixed in chronological order, and they are mapped onto the vertical (amplitude) axis. The resulting patterns can be obtained reading the labels in the vertical axis from bottom to top (in the direction of the increasing amplitude). This method is used by Refs. 2, 3, 33, and 56, among others.

As we mentioned previously, the main goal of these methodologies is to define a set of symbols $\tilde{\pi}^{(D)}$ with an unambiguous rule for mapping between real valued embedded vectors $\mathbf{X}^{(D)}$ to these symbols, permuting the ranks according to time, or permuting the time indexes according to the ranks that have the same value. Figures 1 and 2 reveal that the mappings differ in two out of six symbols in the case $D=3$. As the embedding dimension (pattern length) $D$ increases so does the differences sequence of apparition between both mappings. While these have no effect in the the calculation of Information Theory based quantifiers of global character, like Shannon entropy (Permutation Entropy), they play an important role when the symbolization alphabet needs to be extended in order to treat time series with ties, ${ }^{56}$ or when using quantifiers of local character like Fisher's information measure (Permutation Fisher Information ${ }^{8,59,60}$ ).

\section{PERMUTATION SHANNON ENTROPY}

Entropy is a basic quantity with multiple field-specific interpretations: for instance, it has been associated with disorder, state-space volume, and lack of information..$^{61}$ When dealing with information content, the Shannon entropy is often considered as the foundational and most natural one. ${ }^{62,63}$

Let $P=\left\{p_{j} ; j=1, \ldots, N\right\}$ with $\sum_{j=1}^{N} p_{j}=1$, be a discrete probability distribution function (PDF), with $N$ the number of possible states of the system under study. The Shannon's logarithmic information measure reads

$$
\mathrm{S}[P]=-\sum_{j=1}^{N} p_{j} \ln \left[p_{j}\right] .
$$

This can be regarded to as a measure of the uncertainty associated (information) with the physical process described by $P$. For instance, if $\mathrm{S}[P]=\mathrm{S}_{\min }=0$, we are in position to predict with complete certainty which of the possible outcomes $j$ will actually take place. Our knowledge of the underlying process described by the PDF is maximal in this instance. In contrast, our knowledge is minimal for a uniform distribution $P_{e}=\left\{p_{j}=1 / N\right\}$ since every outcome exhibits the same probability of occurrence, and the uncertainty is maximal, $\mathrm{S}\left[P_{e}\right]=$ $\mathrm{S}_{\max }=\ln N$. We define a "normalized" Shannon entropy, $0 \leq$ $H \leq 1$, as $H[P]=\mathrm{S}[P] / \mathrm{S}_{\max }$.
123

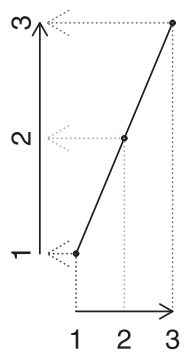

132

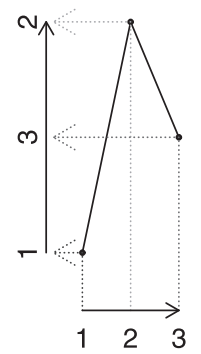

213

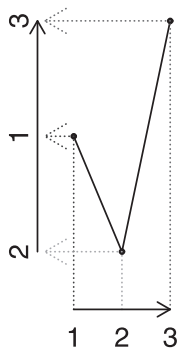

231

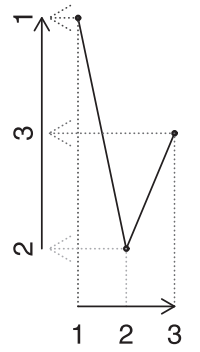

312

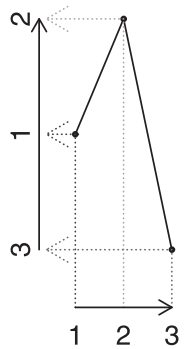

321

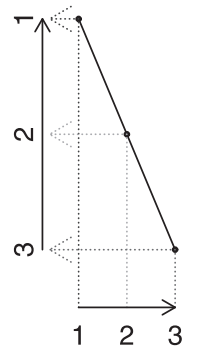

FIG. 2. Chronological index permutation mapping: All symbols for $D=3$ are shown. This order based alphabet simply maps each value $x_{i}$ in the corresponding embedded vector $\mathbf{X}_{t}^{(D)}$ ordering its time index $1 \leq i \leq D$ according to the increasing amplitude of each $x_{i}$. It can be seen that the indexes of the time axis are fixed in chronological order, and they are mapped onto the vertical (amplitude) axis. For each vector $\mathbf{X}_{t}^{(3)}=\left(x_{t}, x_{t+1}, x_{t+2}\right)$, the resulting pattern $\tilde{\pi}^{(3)}$ can be obtained reading the labels in the vertical axis from the bottom to the top (in the direction of the increasing amplitude). 
We use the Bandt-Pompe symbolization methodology for associating with a given time series $\mathcal{X}$, of length $T$, a probability distribution of patterns. That is, we evaluate for an embedding dimension $D$ (pattern length) and a time lag $\tau$, the corresponding vectors $\mathbf{X}_{t}^{(D, \tau)}$ and their associated permutation patterns $\tilde{\pi}^{(D)}$. Their relative frequencies can be naturally computed according to the number of times this particular order sequence (pattern) is found in the time series, divided by the total number of sequences:

$$
p\left(\tilde{\pi}^{(D)}\right)=\frac{\begin{array}{c}
\#\left\{t \mid t \leq T-(D-1) \tau ; \mathbf{X}_{t}^{(D, \tau)}\right. \\
\text { has ordinal pattern } \left.\tilde{\pi}^{(D)}\right\}
\end{array}}{T-(D-1) \tau},
$$

where \# denotes cardinality. We, thus, obtain an ordinal pattern probability distribution $\Pi=\left\{p\left(\tilde{\pi}_{k}^{(D)}\right), k=1, \ldots, D\right.$ ! $\}$ from the time series. The (normalized) Shannon entropy, evaluated with this ordinal pattern probability distribution, is called the permutation (normalized) Shannon entropy.

In the case of time series given in (3), using the rank permutation mapping [see Eq. (4)] we have: $p\left(\tilde{\pi}_{1}^{(3)}\right)=p(123)=2 / 5, \quad p\left(\tilde{\pi}_{2}^{(3)}\right)=p(132)=0, \quad p\left(\tilde{\pi}_{3}^{(3)}\right)=$ $p(213)=1 / 5, p\left(\tilde{\pi}_{4}^{(3)}\right)=p(231)=2 / 5, p\left(\tilde{\pi}_{5}^{(3)}\right)=p(312)=$ $0, p\left(\tilde{\pi}_{6}^{(3)}\right)=p(321)=0$; but if we use the chronological index permutation mapping [see Eq. (5)] we have: $p\left(\tilde{\pi}_{1}^{(3)}\right)=p(123)=2 / 5, \quad p\left(\tilde{\pi}_{2}^{(3)}\right)=p(132)=0, \quad p\left(\tilde{\pi}_{3}^{(3)}\right)=$ $p(213)=1 / 5, \quad p\left(\tilde{\pi}_{4}^{(3)}\right)=p(231)=0, \quad p\left(\tilde{\pi}_{5}^{(3)}\right)=p(312)=$ $2 / 5, p\left(\tilde{\pi}_{6}^{(3)}\right)=p(321)=0$. However, the corresponding permutation Shannon entropy is $S^{(D=3)}[\Pi] \approx 1.0549$ and the permutation normalized Shannon-entropy is $H^{(D=3)}[\Pi] \approx$ 0.6554 , regardless the mapping. A graphical representation of all possible patterns corresponding to $D=3,4$, and 5 can be found in Fig. 2 of Parlitz et al. ${ }^{33}$

\section{TIME SERIES WITH TIES AND THE MAPPINGS TO A SYMBOLIC ALPHABET}

The condition $P\left(x_{t_{1}}=x_{t_{2}}\right)=0 \forall t_{1} \neq t_{2}$ is satisfied assuming that the time series $\mathcal{X}$ under analysis corresponds to a continuous process. With this, the embedding vectors have $D$ unique values (no ties), and both mappings mentioned in Sec. II lead to the same permutation Shannon entropy.

That condition may not be satisfied in several real-world time series, so a substantial amount of embedding vectors $\mathbf{X}_{t}^{(D, \tau)}$ may have equal component values and the mapping $\mathbf{X}_{t}^{(D, \tau)} \mapsto \tilde{\pi}^{(D)}$ cannot be made in neither rank or chronological index permutation mappings (cf. Section III). A number of methodologies have been proposed to handle with time series with ties.

There are two basic strategies for dealing with ties. The first one assumes that the process is indeed continuous so the patterns with ties are in fact missing data deriving from non-tied values patterns wrongly observed. The second one makes no such assumption and extends the alphabet in order to ignore the restriction $\left\{x_{i} \neq x_{j} \forall i \neq j\right\}$ for the components of the vector $\mathbf{X}_{t}^{(D, \tau)}$, mapping equal values to the same symbol, this is the methodology proposed by Bian et $a l .{ }^{56}$ These extended alphabets have more patterns than $D$ ! and vary accordingly if they were constructed based on the time order or the rank symbolization. In consequence, Bian's approach has a different physical interpretation and can not be considered as a generalized Bandt-Pompe symbolization. Clearly, the Shannon entropy evaluated with this extended symbolization does not reach its maximum value for a totally uncorrelated random signal (white noise), as for the Bandt and Pompe symbolization. Then, we only consider the first option for the treatment of time series with ties.

For all the following examples, the Chronological Index Permutation based alphabet will be used. Let us consider, as a numerical example, the following time series with ties:

$$
\begin{aligned}
\mathcal{X} & \equiv\left\{x_{t}\right\}_{t=1}^{T=22} \\
& =\{2,5,1,2,7,1,1,3,1,2,4,5,1,3,2,4,4,2,2,1,0\} .
\end{aligned}
$$

For $D=3$ and $\tau=1$, we have $M=19$ vectors $\mathbf{X}^{(D=3)}$. Denoting $n^{\star}$ the number of which have equal components values, we have $n^{\star}=7$, which amounts to approximately $36.84 \%$ of the vectors; call this percentage $\eta$.

As we mention previously, if one assumes that the time series under study is a realization of a continuous data generator process, the repeated values are in fact missing data deriving from non-tied values patterns wrongly observed. Missing data are a common problem in all types of research fields and there are various methods for handling them that can be divided into two major strategies: the first is to eliminate all the incomplete observations, and the second one is to impute the missing data with a suitable value. ${ }^{64}$

\section{A. Complete cases}

This method was originally suggested by Bandt and Pompe in their original article $^{2}$ and it is analogue to a complete case analysis in Statistics. It consists in eliminating the vectors (patterns) with ties. For example, for $D=3$ and the previous time series $\mathcal{X}=\left\{x_{t}\right\}_{t=1}^{T=22}$, see (8), the vectors $\mathbf{X}_{5}^{(3)}=(7,1,1), \mathbf{X}_{6}^{(3)}=(1,1,3), \mathbf{X}_{7}^{(3)}=(1,3,1), \mathbf{X}_{15}^{(3)}=$ $(2,4,4), \mathbf{X}_{16}^{(3)}=(4,4,2), \mathbf{X}_{17}^{(3)}=(4,2,2)$, and $\mathbf{X}_{18}^{(3)}=(2,2,1)$ are eliminated. The remaining 12 vectors are mapped to the following permutation patterns: $\mathbf{X}_{1}^{(3)}, \mathbf{X}_{4}^{(3)}, \mathbf{X}_{11}^{(3)} \mapsto \tilde{\pi}^{(3)}=$ $312, \mathbf{X}_{2}^{(3)}, \mathbf{X}_{8}^{(3)}, \mathbf{X}_{12}^{(3)} \mapsto \tilde{\pi}^{(3)}=231, \mathbf{X}_{3}^{(3)}, \mathbf{X}_{9}^{(3)}, \mathbf{X}_{10}^{(3)} \mapsto \tilde{\pi}^{(3)}=$ $123, \mathbf{X}_{13}^{(3)} \mapsto \tilde{\pi}^{(3)}=132, \mathbf{X}_{14}^{(3)} \mapsto \tilde{\pi}^{(3)}=231$, and $\mathbf{X}_{19}^{(3)} \mapsto$ $\tilde{\pi}^{(3)}=321$. Then, the probabilities associated with the six patterns $p\left(\tilde{\pi}_{k}^{(3)}\right), k=1, \ldots, 3$ ! are computed using Eq. (7); see Fig. 3 for a representation of the corresponding PDF, and Tables I and II for numerical values comparison. The permutation Shannon entropy is $S^{(D=3)}[\Pi] \approx 1.6609$, and the normalized permutation Shannon entropy is $H^{(D=3)}[\Pi] \approx$ 0.9270 .

\section{B. Time ordered imputation}

This is one of the most used techniques to deal with repeated values. It is employed by Refs. 2, 3, 11, 33, 52, and 65 , among others. It simply states that if $x_{t_{1}}=x_{t_{2}}$ and $t_{1}<t_{2}$ then $x_{t_{1}}<x_{t_{2}}$. Following the previous example, vectors without equal values do not change in their mappings; now those vectors with equal components are included and, according with the rule they are mapped to: $\mathbf{X}_{16}^{(3)} \mapsto \tilde{\pi}^{(3)}=312$, $\mathbf{X}_{5}^{(3)}, \mathbf{X}_{17}^{(3)} \mapsto \tilde{\pi}^{(3)}=231, \quad \mathbf{X}_{6}^{(3)}, \mathbf{X}_{15}^{(3)} \mapsto \tilde{\pi}^{(3)}=123, \quad \mathbf{X}_{7}^{(3)} \mapsto$ 

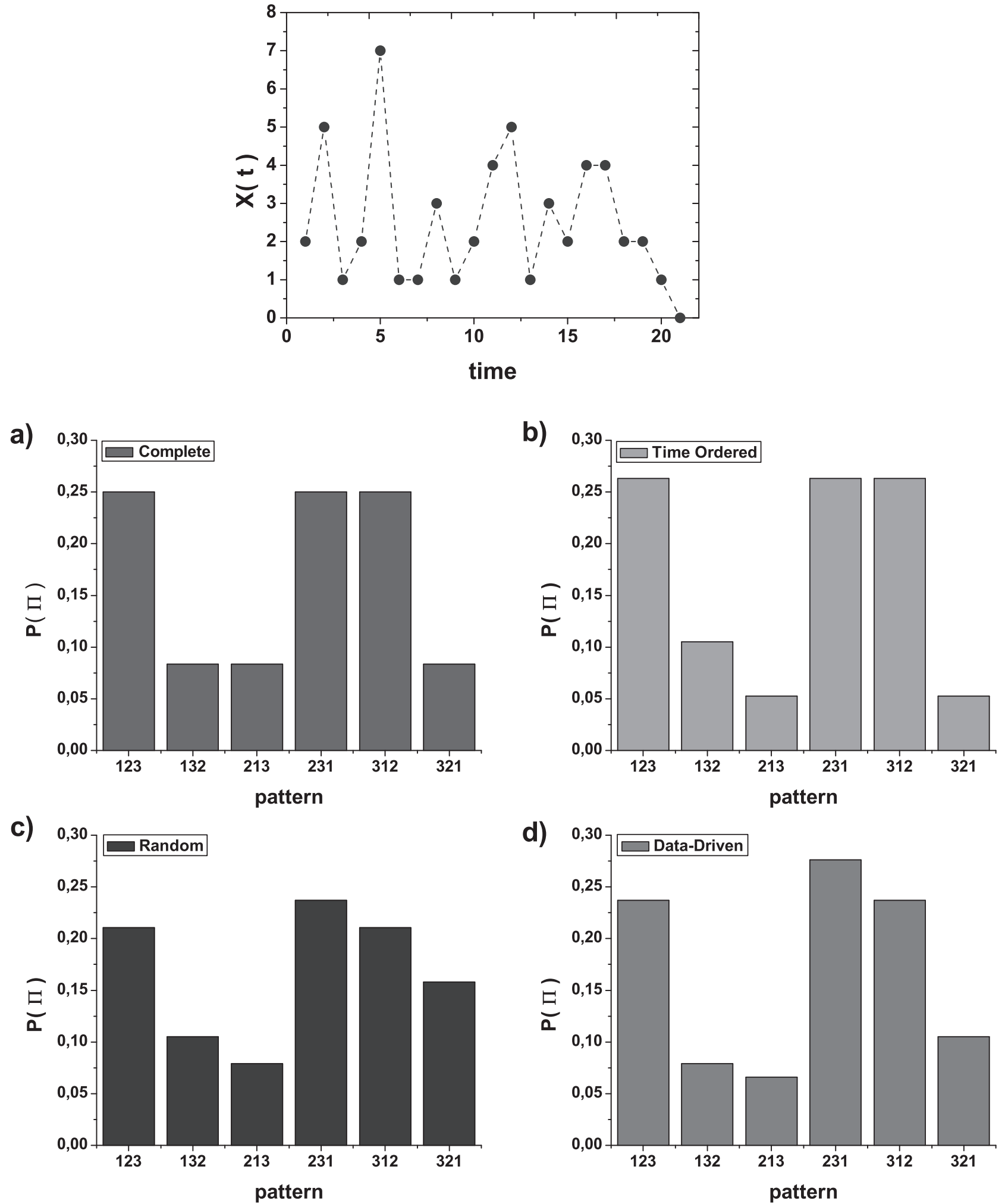

FIG. 3. Upper panel: Time-series with ties [see Eq. (8)], lower panel: the associated Bandt-Pompe PDFs, with $D=3$ and $\tau=1$, obtained with the different approaches for the treatment of the ties values corresponding to the $N=3 !=6$ permutation patterns $\tilde{\pi}^{(3)}$. The corresponding permutation normalized Shannon entropies are (a) $H^{(D=3)}[\Pi] \approx 0.9270$ for Complete BP-PDF; (b) $H^{(D=3)}[\Pi] \approx 0.8935$ for Time Ordered BP-PDF; (c) $H^{(D=3)}[\Pi] \approx 0.9633$ for Random BP-PDF and (d) $H^{(D=3)}[\Pi] \approx 0.9232$ for Data-Driven BP-PDF. 
TABLE I. Reconstructed vectors with embedding dimension $D=3$ and time dimension $\tau=1$ for time series with ties $\mathcal{X}=\left\{x_{t}\right\}_{t=1}^{T=22}$, given by Eq. (8). For the $M=19$ vectors $\mathbf{X}^{(D=3)}, n^{\star}=7$ of them present equal component values representing the $\eta=36.84 \%$ of the reconstructed vectors. For each reconstructed vector $\mathbf{X}^{(D=3)}$, the corresponding permutation mapping (chronological time ordering) $\tilde{\pi}^{(D=3)}$ following the imputation methodology for the treatment of tied values: Complete, Time Ordered, Random, and Data-Driven are given (for details, see Sec. IV).

\begin{tabular}{|c|c|c|c|c|c|c|c|}
\hline \multirow{3}{*}{$t$} & \multirow{3}{*}{$\mathbf{X}_{t}^{(D=3)}$} & \multicolumn{6}{|c|}{ Imputation method: } \\
\hline & & \multirow{2}{*}{$\begin{array}{c}\text { Complete } \\
\tilde{\pi}^{(D=3)}\end{array}$} & \multirow{2}{*}{$\begin{array}{c}\text { Time Ordered } \\
\tilde{\pi}^{(D=3)}\end{array}$} & \multicolumn{2}{|c|}{ Random } & \multicolumn{2}{|r|}{ Data-Driven } \\
\hline & & & & $\tilde{\pi}^{(D=3)}$ & With $\omega$ & $\tilde{\pi}^{(D=3)}$ & With $\omega^{*}$ \\
\hline 1 & $(2,5,1)$ & 312 & 312 & 312 & 1 & 312 & 1 \\
\hline 2 & $(5,1,2)$ & 231 & 231 & 231 & 1 & 231 & 1 \\
\hline 3 & $(1,2,7)$ & 123 & 123 & 123 & 1 & 123 & 1 \\
\hline 4 & $(2,7,1)$ & 312 & 312 & 312 & 1 & 312 & 1 \\
\hline \multirow[t]{2}{*}{5} & $(7,1,1)$ & $\ldots$ & To 231 & To 231 & $1 / 2$ & To 231 & $p(231) /\{p(231)+p(321)\}=3 / 4$ \\
\hline & & & & To 321 & $1 / 2$ & To 321 & $p(321) /\{p(231)+p(321)\}=1 / 4$ \\
\hline \multirow[t]{2}{*}{6} & $(1,1,3)$ & $\ldots$ & To 123 & To 123 & $1 / 2$ & To 123 & $p(123) /\{p(123)+p(213)\}=3 / 4$ \\
\hline & & & & To 213 & $1 / 2$ & To 213 & $p(213) /\{p(123)+p(213)\}=1 / 4$ \\
\hline \multirow[t]{2}{*}{7} & $(1,3,1)$ & $\ldots$ & To 132 & To 132 & $1 / 2$ & To 132 & $p(132) /\{p(123)+p(231)\}=1 / 4$ \\
\hline & & & & To 231 & $1 / 2$ & To 231 & $p(231) /\{p(123)+p(231)\}=3 / 4$ \\
\hline 8 & $(3,1,2)$ & 231 & 231 & 231 & 1 & 231 & 1 \\
\hline 9 & $(1,2,4)$ & 123 & 123 & 123 & 1 & 123 & 1 \\
\hline 10 & $(2,4,5)$ & 123 & 123 & 123 & 1 & 123 & 1 \\
\hline 11 & $(4,5,1)$ & 312 & 312 & 312 & 1 & 312 & 1 \\
\hline 12 & $(5,1,3)$ & 231 & 231 & 231 & 1 & 231 & 1 \\
\hline 13 & $(1,3,2)$ & 132 & 132 & 132 & 1 & 132 & 1 \\
\hline 14 & $(3,2,4)$ & 213 & 213 & 213 & 1 & 213 & 1 \\
\hline \multirow[t]{2}{*}{15} & $(2,4,4)$ & $\ldots$ & To 123 & To 123 & $1 / 2$ & To 123 & $p(123) /\{p(123)+p(132)\}=3 / 4$ \\
\hline & & & & To 132 & $1 / 2$ & To 132 & $p(132) /\{p(123)+p(132)\}=1 / 4$ \\
\hline \multirow[t]{2}{*}{16} & $(4,4,2)$ & $\ldots$ & To 312 & To 312 & $1 / 2$ & To 312 & $p(312) /\{p(312)+p(321)\}=3 / 4$ \\
\hline & & & & To 321 & $1 / 2$ & To 321 & $p(321) /\{p(312)+p(321)\}=1 / 4$ \\
\hline \multirow[t]{2}{*}{17} & $(4,2,2)$ & $\ldots$ & To 231 & To 231 & $1 / 2$ & To 231 & $p(231) /\{p(231)+p(321)\}=3 / 4$ \\
\hline & & & & To 321 & $1 / 2$ & To 321 & $p(321) /\{p(231)+p(321)\}=3 / 4$ \\
\hline \multirow[t]{2}{*}{18} & $(2,2,1)$ & $\ldots$ & 312 & To 312 & $1 / 2$ & To 312 & $p(312) /\{p(312)+p(321)\}=3 / 4$ \\
\hline & & & & To 321 & $1 / 2$ & To 321 & $p(321) /\{p(312)+p(321)\}=1 / 4$ \\
\hline 19 & $(2,1,0)$ & 321 & 321 & 213 & 1 & 213 & 1 \\
\hline
\end{tabular}

$\tilde{\pi}^{(3)}=132$, and $\mathbf{X}_{18}^{(3)} \mapsto \tilde{\pi}^{(3)}=321$. The patterns probabilities $p\left(\tilde{\pi}_{k}^{(3)}\right), k=1, \ldots, 3$ ! are computed again using Eq. (7), but now over the 19 vectors. Figure 3 shows the obtained PDF; see also Table II for numerical values comparison. The permutation Shannon entropy is $S^{(D=3)}[\Pi] \approx 1.6001$, and the normalized permutation Shannon entropy is $H^{(D=3)}[\Pi] \approx$ 0.8935 .

\section{Random imputation}

Bandt and Pompe, in their seminal paper, ${ }^{2}$ recommend to numerically break equalities by adding small random perturbations, e.g., observational noise. The amplitude of this perturbation should be sufficiently small to not modify the ordinal relations in the vector component, except for those vectors component which have equal values.

TABLE II. Associated probabilities to the six permutation patterns $\tilde{\pi}_{k}^{(3)}$ evaluated for the time series $\mathcal{X}=\left\{x_{t}\right\}_{t=1}^{T=22}$, given in (8), with $D=3$ and $\tau=1$, when the four different imputation methodologies (see Sec. IV) were considered for the case of vectors $\mathbf{X}_{t}^{(D)}$ present equal component values (see Table I). In the last row, we check that the sum over all probabilities patterns (columns) is one.

\begin{tabular}{|c|c|c|c|c|c|}
\hline \multicolumn{2}{|c|}{ Permutation pattern } & \multicolumn{4}{|c|}{ Obtained probability with imputation method } \\
\hline$k$ & $\tilde{\pi}_{k}^{(D=3)}$ & Complete & Time Ordered & Random & Data-Driven \\
\hline 1 & 123 & $3 / 12$ & $5 / 19$ & $4 / 19$ & $18 / 76$ \\
\hline 2 & 132 & $1 / 12$ & $2 / 19$ & $2 / 19$ & $6 / 76$ \\
\hline 3 & 213 & $1 / 12$ & $1 / 19$ & $3 / 38$ & $5 / 76$ \\
\hline 4 & 231 & $3 / 12$ & $5 / 19$ & $9 / 38$ & $21 / 76$ \\
\hline 5 & 312 & $3 / 12$ & $5 / 19$ & $4 / 19$ & $18 / 76$ \\
\hline \multirow[t]{2}{*}{6} & 321 & $1 / 12$ & $1 / 19$ & $3 / 19$ & $8 / 76$ \\
\hline & $\sum_{k}$ & 1 & 1 & 1 & 1 \\
\hline
\end{tabular}


In our example, the vector $\mathbf{X}_{1}^{(3)}=(2,5,1)$ will be mapped to the ordinal pattern $\tilde{\pi}^{(3)}=(312)$ with weight $\omega=1$, since it does not have equal values. A vector with two equal components, as $\mathbf{X}_{5}^{(3)}=(7,1,1)$, should be mapped either to $\tilde{\pi}^{(3)}=$ (231) or to $\tilde{\pi}^{(3)}=(321)$, with weight $\omega=1 / 2$ each. These are the only possible choices, since the perturbation cannot alter the first component of the vector. Six symbols may appear after perturbation if all three values are equal, e.g $\mathbf{X}_{k}^{(3)}=(7,7,7)$, each with weight $\omega=1 / 6$. We now compute the resulting pattern probability $p\left(\tilde{\pi}^{(3)}\right), k=1, \ldots, 3$ ! over all the 19 vectors but considering the weights. As before, the obtained PDF is shown in Fig. 3 and, numerical values are given in Table II, with permutation Shannon entropy $\mathrm{S}^{(D=3)}[\Pi] \approx 1.7261$ and normalized permutation Shannon entropy $H^{(D=3)}[\Pi] \approx 0.9633$.

\section{Data-driven imputation}

Random Imputation suggests that, regardless the time series, as patterns with equal values are the result of a coarse observation of patterns without ties in the original series, they should be mapped onto symbols corresponding to those original patterns with the same probabilistic weight. In many situations, simple procedures for handling missing data (such as complete cases or random imputation) produce biased results, ${ }^{64}$ and more sophisticated techniques lead to much better results. Such techniques, impute missing data by a value that is predicted using the subject's other known characteristics.

We propose a method which is similar to the random imputation but, instead of adding a random perturbation that maps with equal probability to each suitable symbol, these probabilities are originated with a previously known PDF, and are not necessarily equal. The PDF proposed as a prior distribution is the one resulting from computing $p\left(\tilde{\pi}_{k}^{(D)}\right), k=$ $1, \ldots, D$ ! for the complete cases (see Subsection IV A). We call this technique "Data-Driven," as it uses the available information to fill the gaps produced by repeated values.

The procedure consists of eight steps:

1. Define the embedding dimension $D$, that leads to the all possible permutation patterns $\tilde{\pi}_{k}^{(D)}$ with $k=1, \ldots, D$ !.

2. Map all the reconstructed vectors $\mathbf{X}_{t}^{(D)}$ to their correspondent permutation pattern $\tilde{\pi}_{k}^{(D)}$ using chronological order (see Subsection II B).

3. If there is any ties in $\mathbf{X}_{t}^{(D)}$ for any $t$, eliminate the vector (Subsection IV A).

4. Calculate the permutation patterns probabilities $p\left(\tilde{\pi}_{k}^{(D)}\right), k=1, \ldots, D$ ! using Eq. (7).

5. Repeat the procedure of mapping every $\mathbf{X}_{t}^{(D)}$, but do not eliminate the vectors with repeated values.

6. If the vector $\mathbf{X}_{t}^{(D)}$ has not equal components, map it to pattern $\tilde{\pi}^{(D)}$ with weight $\omega^{*}\left(\tilde{\pi}^{(D)}\right)=1$.

7. If the vector $\mathbf{X}_{t}^{(D)}$ has equal components, break ties by adding small perturbations, similarly to random imputation (see Subsection IV C). Identify all the $m$ suitable pattern $\tilde{\pi}_{m}^{(D)}$ and assign each a weight $\omega^{*}\left(\tilde{\pi}_{m}^{(D)}\right)=$ $p\left(\tilde{\pi}_{m}^{(D)}\right) /\left\{\sum_{m} p\left(\tilde{\pi}_{m}^{(D)}\right)\right\}$. Note: the probabilities $p\left(\tilde{\pi}^{(D)}\right)$ are those obtained in step 4.
8. Calculate the resulting pattern probability $p^{*}\left(\tilde{\pi}^{(D)}\right), k=$ $1, \ldots, D$ ! over all the reconstructed vectors but now considering the associated weight of apparition $\left(\omega^{*}\left(\tilde{\pi}^{(D)}\right)=\right.$ 1 for vectors without ties, and $\omega^{*}\left(\tilde{\pi}^{(D)}\right) \neq 1$ otherwise $)$.

Figure 3 and Table II show the obtained PDF and numerical values for the previous example, which lead to permutation Shannon entropy $\mathrm{S}^{(D=3)}[\Pi] \approx 1.6541$ and normalized permutation Shannon entropy $H^{(D=3)}[\Pi] \approx 0.9232$.

It is noteworthy that Random Imputation may overestimate the entropy, as it adds random noise to the series, and masks forbidden patters by inducing those missing patterns to appear, concealing this dynamical property characteristic of chaotic dynamics. The probabilities used in Datadriven imputation, instead, are derived from the Complete Cases methodology, taking into account of the structure of the time series, preserving, thus, the missing or forbidden patterns.

All possible patterns for $D=3$ are shown in the first column of Table III, and their mapping for every methodology is presented. The above described procedures for the imputation of permutation patterns starting with the reconstructed vectors can be easily extended to patterns with embedding dimension $D>3$.

\section{NUMERICAL RESULTS AND DISCUSSION}

In this section, we apply the presented strategies for mapping time series with ties to study the decimal expansion of irrational numbers and time series data from chaotic maps recorded with low precision. Their performance is also analyzed.

\section{A. Decimal expansion of irrational numbers}

We are interested in the evaluation of the randomness of the decimal expansion of irrational numbers using Permutation Entropy. For that propose, we analyze the Bandt-Pompe PDF of the temporal sequences obtained by picking the first $10^{6}$ digits of the decimal expansion of the irrational numbers $\pi, e$, and $\sqrt{2}$. In particular, for these three irrational numbers we consider the time series with length $T=5 \times 10^{3}, 10^{4}, 10^{5}$, and $10^{6}$ of the first digits and evaluate the Bandt-Pompe PDF with embedding dimensions $D=3,4,5,6$, and time lag $\tau=1$.

Tables IV-VI present the results for $\pi, e$, and $\sqrt{2}$, respectively. We reported the number of reconstructed vectors of dimension $D$ that present equal component values, denoted by $n^{\star}$, the percent ratio of these vectors in relation to the total number of possible vectors, $\eta$, for the time series length $T$ and the obtained values for the normalized permutation Shannon entropy $H^{(D)}[\Pi]$, evaluated with BP-PDF using Complete, Time Ordered, and Data-Driven imputation methods, as well as the number of forbidden/missing patterns (NFMP) for each case. Note that even when $n^{\star}$ is an increasing function of the time series length $T$ and the embedding dimension $D$, the percentage ratio $\eta$ is almost independent of $T$ for a fixed $D$. Moreover, as can be seen from the results displayed in Tables IV-VI, $\eta$ presents very similar values for the three irrational 
TABLE III. All possible patterns for $D=3$ are shown in the first column, and their mapping for every methodology are presented.

\begin{tabular}{|c|c|c|c|c|c|c|}
\hline \multirow{3}{*}{ Pattern } & \multicolumn{6}{|c|}{ Imputation method: } \\
\hline & \multirow{2}{*}{$\begin{array}{l}\text { Complete } \\
\tilde{\pi}^{(D=3)}\end{array}$} & \multirow{2}{*}{$\begin{array}{c}\text { Time Ordered } \\
\qquad \tilde{\pi}^{(D=3)}\end{array}$} & \multicolumn{2}{|c|}{ Random } & \multicolumn{2}{|r|}{ Data-Driven } \\
\hline & & & $\tilde{\pi}^{(D=3)}$ & With $\omega$ & $\tilde{\pi}^{(D=3)}$ & With $\omega^{*}$ \\
\hline & $\ldots$ & To 123 & To 123 & $\omega=1 / 6$ & To 123 & $\omega^{*}=p(123)$ \\
\hline & & & To 132 & $\omega=1 / 6$ & To 132 & $\omega^{*}=p(132)$ \\
\hline & & & To 213 & $\omega=1 / 6$ & To 213 & $\omega^{*}=p(213)$ \\
\hline & & & To 231 & $\omega=1 / 6$ & To 231 & $\omega^{*}=p(231)$ \\
\hline & & & To 312 & $\omega=1 / 6$ & To 312 & $\omega^{*}=p(312)$ \\
\hline & & & To 321 & $\omega=1 / 6$ & To 321 & $\omega^{*}=p(321)$ \\
\hline & $\ldots$ & To 132 & To 132 & $\omega=1 / 2$ & To 132 & $\omega^{*}=p(132) /\{p(132)+p(312)\}$ \\
\hline & & & To 312 & $\omega=1 / 2$ & To 312 & $\omega^{*}=p(312) /\{p(132)+p(312)\}$ \\
\hline & $\ldots$ & To 123 & To 123 & $\omega=1 / 2$ & To 123 & $\omega^{*}=p(123) /\{p(123)+p(213)\}$ \\
\hline & & & To 213 & $\omega=1 / 2$ & To 213 & $\omega^{*}=p(213) /\{p(123)+p(213)\}$ \\
\hline & $\ldots$ & To 123 & To 123 & $\omega=1 / 2$ & To 123 & $\omega^{*}=p(123) /\{p(123)+p(132)\}$ \\
\hline & & & To 132 & $\omega=1 / 2$ & To 132 & $\omega^{*}=p(132) /\{p(123)+p(132)\}$ \\
\hline & 123 & 123 & 123 & $\omega=1$ & 123 & $\omega^{*}=1$ \\
\hline & 132 & 132 & 132 & $\omega=1$ & 132 & $\omega^{*}=1$ \\
\hline & $\ldots$ & To 213 & To 213 & $\omega=1 / 2$ & To 213 & $\omega^{*}=p(213) /\{p(213)+p(231)\}$ \\
\hline & & & To 231 & $\omega=1 / 2$ & To 231 & $\omega^{*}=p(231) /\{p(213)+p(231)\}$ \\
\hline & 213 & 213 & 213 & $\omega=1$ & 213 & $\omega^{*}=1$ \\
\hline & $\ldots$ & To 312 & To 312 & $\omega=1 / 2$ & To 312 & $\omega^{*}=p(312) /\{p(312)+p(321)\}$ \\
\hline & & & To 321 & $\omega=1 / 2$ & To 321 & $\omega^{*}=p(321) /\{p(312)+p(321)\}$ \\
\hline & 231 & 231 & 231 & $\omega=1$ & 231 & $\omega^{*}=1$ \\
\hline & $\ldots$ & To 231 & To 231 & $\omega=1 / 2$ & To 231 & $\omega^{*}=p(231) /\{p(231)+p(321)\}$ \\
\hline & & & To 321 & $\omega=1 / 2$ & To 321 & $\omega^{*}=p(321) /\{p(231)+p(321)\}$ \\
\hline & 312 & 312 & 312 & $\omega=1$ & 312 & $\omega^{*}=1$ \\
\hline & 321 & 321 & 321 & $\omega=1$ & 321 & $\omega^{*}=1$ \\
\hline
\end{tabular}

numbers. This can be linked to the fact that decimal expansion consists of the digits from 0 to 9 . It is noteworthy that the Time Ordered Shannon entropy is significantly larger than that obtained by the Data-Driven technique. This is an effect of large vectors $(D=6)$ of values in the set $\{0,1, \ldots, 9\}$ which will are likely to have repetitions. The latter technique tries to fill more than $80 \%$ of observations with only $20 \%$ of the data without repeated values.

The values of $H^{(D)}[\Pi]$ evaluated with BP-PDF following the time order imputation suggest the presence of temporal complex structures in the data, for increasing values of
$D \geq 4$, regardless the time series length $T$. Remarkably, these values are quite similar for the three decimal expansion of irrational numbers. This effect, as shown by Zunino et al. ${ }^{55}$ is due to ties. The same operator, when applied to Data-Driven imputed data, does not show any kind of correlation structure, indicating that the decimal expansion of the irrational numbers are compatible with uncorrelated random behavior in agreement with the results obtained by Luque et al. ${ }^{66}$ using Horizontal Visibility Graphs. Note also that similar results are obtained when using BP-PDF with complete imputation, however, in this case, we have a strong reduction of the number 
TABLE IV. Results for the decimal expansion of $\pi$. We reported the number of reconstructed vectors of dimension $D$ that present equal component values, $n^{\star}$, the percent ratio of these vectors in relation to the total number of possible vectors, $\eta$, for the time series length $T$ and the obtained values for the permutation normalized Shannon entropy $H^{(D)}[\Pi]$, evaluated with BP-PDF using the imputation method: Complete, Time Ordered, and Data-Driven, as well as the number of forbidden/missing patterns (NFMP) in each case.

\begin{tabular}{|c|c|c|c|c|c|c|c|c|c|}
\hline \multicolumn{10}{|c|}{ Decimal expansion for $\pi$} \\
\hline \multirow[t]{2}{*}{$T$} & \multirow[t]{2}{*}{$D$} & \multirow[t]{2}{*}{$n^{\star}$} & \multirow[t]{2}{*}{$\eta[\%]$} & \multicolumn{2}{|c|}{ BP-PDF Complete } & \multicolumn{2}{|c|}{ BP-PDF Time Ordered } & \multicolumn{2}{|c|}{ BP-PDF Data-Driven } \\
\hline & & & & $H[\Pi]$ & $N F M P$ & $H[\Pi]$ & $N F M P$ & $H[\Pi]$ & NFMP \\
\hline $5 \times 10^{3}$ & 3 & 1332 & 26.64 & 0.999983033 & 0 & 0.993241941 & 0 & 0.999973708 & 0 \\
\hline $1 \times 10^{4}$ & 3 & 2714 & 27.14 & 0.999956462 & 0 & 0.991949033 & 0 & 0.999939272 & 0 \\
\hline $1 \times 10^{5}$ & 3 & 27911 & 27.91 & 0.999988701 & 0 & 0.991990810 & 0 & 0.999991366 & 0 \\
\hline \multirow[t]{2}{*}{$1 \times 10^{6}$} & 3 & 279616 & 27.96 & 0.999999289 & 0 & 0.991634948 & 0 & 0.999999483 & 0 \\
\hline & & & & $H[\Pi]$ & $N F M P$ & $H[\Pi]$ & $N F M P$ & $H[\Pi]$ & NFMP \\
\hline $5 \times 10^{3}$ & 4 & 2423 & 48.46 & 0.999083838 & 0 & 0.990384203 & 0 & 0.999348336 & 0 \\
\hline $1 \times 10^{4}$ & 4 & 4901 & 49.01 & 0.999628671 & 0 & 0.989598352 & 0 & 0.999639847 & 0 \\
\hline $1 \times 10^{5}$ & 4 & 49535 & 49.53 & 0.999950456 & 0 & 0.989752703 & 0 & 0.999966815 & 0 \\
\hline \multirow[t]{2}{*}{$1 \times 10^{6}$} & 4 & 495599 & 49.56 & 0.999993518 & 0 & 0.989473294 & 0 & 0.999995625 & 0 \\
\hline & & & & $H[\Pi]$ & $N F M P$ & $H[\Pi]$ & $N F M P$ & $H[\Pi]$ & $N F M P$ \\
\hline $5 \times 10^{3}$ & 5 & 3470 & 69.40 & 0.990692144 & 0 & 0.985338717 & 0 & 0.994227640 & 0 \\
\hline $1 \times 10^{4}$ & 5 & 6960 & 69.60 & 0.995754267 & 0 & 0.985619302 & 0 & 0.997368567 & 0 \\
\hline $1 \times 10^{5}$ & 5 & 69915 & 69.91 & 0.999518569 & 0 & 0.987094212 & 0 & 0.999703403 & 0 \\
\hline \multirow[t]{2}{*}{$1 \times 10^{6}$} & 5 & 697637 & 69.76 & 0.999954020 & 0 & 0.986937835 & 0 & 0.999973370 & 0 \\
\hline & & & & $H[\Pi]$ & $N F M P$ & $H[\Pi]$ & $N F M P$ & $H[\Pi]$ & $N F M P$ \\
\hline $5 \times 10^{3}$ & 6 & 4241 & 84.82 & 0.912393855 & 259 & 0.972623954 & 12 & 0.948736453 & 53 \\
\hline $1 \times 10^{4}$ & 6 & 8485 & 84.85 & 0.956891046 & 93 & 0.977989801 & 1 & 0.971986907 & 46 \\
\hline $1 \times 10^{5}$ & 6 & 85139 & 85.14 & 0.996105458 & 0 & 0.984057686 & 0 & 0.997787493 & 0 \\
\hline $1 \times 10^{6}$ & 6 & 849015 & 84.90 & 0.999642595 & 0 & 0.984054591 & 0 & 0.999802363 & 0 \\
\hline
\end{tabular}

TABLE V. Results for the decimal expansion of $\pi$ with similar notation of Table IV.

\begin{tabular}{|c|c|c|c|c|c|c|c|c|c|}
\hline \multicolumn{10}{|c|}{ Decimal expansion for $e$} \\
\hline \multirow[t]{2}{*}{$T$} & \multirow[t]{2}{*}{$D$} & \multirow[t]{2}{*}{$n^{\star}$} & \multirow[t]{2}{*}{$\eta[\%]$} & \multicolumn{2}{|c|}{ BP-PDF Complete } & \multicolumn{2}{|c|}{ BP-PDF Time Ordered } & \multicolumn{2}{|c|}{ BP-PDF Data-Driven } \\
\hline & & & & $H[\Pi]$ & $N F M P$ & $H[\Pi]$ & $N F M P$ & $H[\Pi]$ & $N F M P$ \\
\hline $5 \times 10^{3}$ & 3 & 1404 & 28.08 & 0.999908248 & 0 & 0.991383284 & 0 & 0.999938519 & 0 \\
\hline $1 \times 10^{4}$ & 3 & 2834 & 28.34 & 0.999961926 & 0 & 0.991654981 & 0 & 0.999974069 & 0 \\
\hline $1 \times 10^{5}$ & 3 & 28113 & 28.11 & 0.999993385 & 0 & 0.991192180 & 0 & 0.999993201 & 0 \\
\hline \multirow[t]{2}{*}{$1 \times 10^{6}$} & 3 & 279055 & 27.91 & 0.999998017 & 0 & 0.991678797 & 0 & 0.999998368 & 0 \\
\hline & & & & $H[\Pi]$ & $N F M P$ & $H[\Pi]$ & $N F M P$ & $H[\Pi]$ & $N F M P$ \\
\hline $5 \times 10^{3}$ & 4 & 2463 & 49.26 & 0.998405105 & 0 & 0.988365038 & 0 & 0.998945166 & 0 \\
\hline $1 \times 10^{4}$ & 4 & 4962 & 49.62 & 0.999112427 & 0 & 0.988967037 & 0 & 0.999575734 & 0 \\
\hline $1 \times 10^{5}$ & 4 & 49615 & 49.61 & 0.999936691 & 0 & 0.988818611 & 0 & 0.999959068 & 0 \\
\hline \multirow[t]{2}{*}{$1 \times 10^{6}$} & 4 & 494965 & 49.50 & 0.999991569 & 0 & 0.989603095 & 0 & 0.999994710 & 0 \\
\hline & & & & $H[\Pi]$ & $N F M P$ & $H[\Pi]$ & $N F M P$ & $H[\Pi]$ & $N F M P$ \\
\hline $5 \times 10^{3}$ & 5 & 3468 & 69.36 & 0.991670342 & 0 & 0.984363390 & 0 & 0.994357956 & 0 \\
\hline $1 \times 10^{4}$ & 5 & 6977 & 69.77 & 0.985522950 & 0 & 0.995638410 & 0 & 0.997387771 & 0 \\
\hline $1 \times 10^{5}$ & 5 & 69851 & 69.85 & 0.999628731 & 0 & 0.986185864 & 0 & 0.999784352 & 0 \\
\hline \multirow[t]{2}{*}{$1 \times 10^{6}$} & 5 & 696451 & 69.64 & 0.999956277 & 0 & 0.987057780 & 0 & 0.999973223 & 0 \\
\hline & & & & $H[\Pi]$ & $N F M P$ & $H[\Pi]$ & $N F M P$ & $H[\Pi]$ & $N F M P$ \\
\hline $5 \times 10^{3}$ & 6 & 4230 & 84.60 & 0.921487060 & 240 & 0.971655603 & 9 & 0.949427685 & 65 \\
\hline $1 \times 10^{4}$ & 6 & 8489 & 84.89 & 0.960524765 & 85 & 0.977676090 & 0 & 0.972847146 & 52 \\
\hline $1 \times 10^{5}$ & 6 & 85102 & 85.10 & 0.996291932 & 0 & 0.982721401 & 0 & 0.997957587 & 0 \\
\hline $1 \times 10^{6}$ & 6 & 847870 & 84.79 & 0.999628684 & 0 & 0.984099807 & 0 & 0.999791651 & 0 \\
\hline
\end{tabular}

of reconstructed vectors, implying that longer time series are necessary in order to have zero forbidden/missing patterns, as for $D=6$.

\section{B. Chaotic maps time series}

We perform a quantitative assessment of the proposed imputation techniques for low-precision data. Let $\mathcal{X}^{*} \equiv$
$\left\{x_{t}^{*}\right\}_{t=1}^{T}$ and $\mathcal{X} \equiv\left\{x_{t}\right\}_{t=1}^{T}$ be the original and coarse time series, respectively, for which the permutation patterns PDFs (embedding dimension $D$ and time lag $\tau$ ) are $\Pi^{*}$ and $\Pi$, respectively. Consider $\Upsilon$ an operator over a permutation pattern PDF, e.g., the Shannon Entropy. We consider four different ways of performing the mapping $\mathcal{X}$ to a symbolic alphabet, which lead to $\Pi^{(i)}, 1 \leq i \leq 4$ : (1) Complete; (2) Random; (3) Time Ordered, and (4) Data-Driven. 
Our hypothesis is that the operator applied to the best symbolic imputation technique will lead to the value closest to the original value. We thus want to find the technique $i$ for which

$$
\delta^{(i)} \Upsilon=\frac{\left|\Upsilon\left(\Pi^{(i)}\right)-\Upsilon\left(\Pi^{*}\right)\right|}{\Upsilon\left(\Pi^{*}\right)},
$$

is minimum, provided $\Upsilon\left(\Pi^{*}\right) \neq 0$. Note that Eq. (9) quantifies the relative deviation of $\mathcal{X}$ from $\mathcal{X}^{*}$, i.e., it is a measure of the error in which imputation $i$ incurs.

In order to gain a deeper understanding of the underlying mechanics, in the following, we analyze the behavior of $\Upsilon\left(\Pi^{(i)}\right)$ for two different operators $\Upsilon$ : the normalized Permutation Shannon Entropy $(H)$ and the number of forbidden/missing patterns (NFMP), considering embedding dimensions $D=3,4,5,6$, and time lag $\tau=1$.

TABLE VI. Results for decimal expansion of $\sqrt{2}$ with similar notation of Table IV.

\begin{tabular}{|c|c|c|c|c|c|c|c|c|c|}
\hline \multicolumn{10}{|c|}{ Decimal expansion for $\sqrt{2}$} \\
\hline \multirow[t]{2}{*}{$T$} & \multirow[t]{2}{*}{$D$} & \multirow[t]{2}{*}{$n^{\star}$} & \multirow[t]{2}{*}{$\eta[\%]$} & \multicolumn{2}{|c|}{ BP-PDF Complete } & \multicolumn{2}{|c|}{ BP-PDF Time Ordered } & \multicolumn{2}{|c|}{ BP-PDF Data-Driven } \\
\hline & & & & $H[\Pi]$ & $N F M P$ & $H[\Pi]$ & $N F M P$ & $H[\Pi]$ & $N F M P$ \\
\hline $5 \times 10^{3}$ & 3 & 1409 & 28.18 & 0.999747074 & 0 & 0.992042468 & 0 & 0.999801067 & 0 \\
\hline $1 \times 10^{4}$ & 3 & 2716 & 27.16 & 0.999929547 & 0 & 0.992266531 & 0 & 0.999939907 & 0 \\
\hline $1 \times 10^{5}$ & 3 & 27846 & 27.84 & 0.999989334 & 0 & 0.991574371 & 0 & 0.999990756 & 0 \\
\hline \multirow[t]{2}{*}{$1 \times 10^{6}$} & 3 & 280250 & 28.02 & 0.999997983 & 0 & 0.991660462 & 0 & 0.999998337 & 0 \\
\hline & & & & $H[\Pi]$ & $N F M P$ & $H[\Pi]$ & $N F M P$ & $H[\Pi]$ & NFMP \\
\hline $5 \times 10^{3}$ & 4 & 2523 & 50.46 & 0.998989712 & 0 & 0.990023329 & 0 & 0.999251673 & 0 \\
\hline $1 \times 10^{4}$ & 4 & 4904 & 49.04 & 0.999548691 & 0 & 0.990577249 & 0 & 0.999656659 & 0 \\
\hline $1 \times 10^{5}$ & 4 & 49488 & 49.49 & 0.999920019 & 0 & 0.989688015 & 0 & 0.999930773 & 0 \\
\hline \multirow[t]{2}{*}{$1 \times 10^{6}$} & 4 & 496215 & 49.62 & 0.999991682 & 0 & 0.989554250 & 0 & 0.999993704 & 0 \\
\hline & & & & $H[\Pi]$ & $N F M P$ & $H[\Pi]$ & $N F M P$ & $H[\Pi]$ & NFMP \\
\hline $5 \times 10^{3}$ & 5 & 3553 & 71.06 & 0.991309030 & 0 & 0.986108322 & 0 & 0.994527547 & 0 \\
\hline $1 \times 10^{4}$ & 5 & 6925 & 69.25 & 0.996496299 & 0 & 0.987505966 & 0 & 0.997838985 & 0 \\
\hline $1 \times 10^{5}$ & 5 & 69759 & 69.76 & 0.999569331 & 0 & 0.987053875 & 0 & 0.999713953 & 0 \\
\hline \multirow[t]{2}{*}{$1 \times 10^{6}$} & 5 & 698094 & 69.81 & 0.999960755 & 0 & 0.986971074 & 0 & 0.999976074 & 0 \\
\hline & & & & $H[\Pi]$ & $N F M P$ & $H[\Pi]$ & $N F M P$ & $H[\Pi]$ & $N F M P$ \\
\hline $5 \times 10^{3}$ & 6 & 4296 & 85.92 & 0.909654949 & 273 & 0.973938788 & 12 & 0.949348636 & 52 \\
\hline $1 \times 10^{4}$ & 6 & 8471 & 84.71 & 0.959981670 & 86 & 0.980471033 & 1 & 0.972931263 & 59 \\
\hline $1 \times 10^{5}$ & 6 & 84955 & 84.95 & 0.996167477 & 0 & 0.983814885 & 0 & 0.997780319 & 0 \\
\hline $1 \times 10^{6}$ & 6 & 849624 & 84.96 & 0.999609416 & 0 & 0.984012788 & 0 & 0.999792633 & 0 \\
\hline
\end{tabular}

TABLE VII. Results for the Logistic Map with $\rho=4$. The values reported as Original Data, are those reported in Ref. [49] and the values reported as Truncated Data, are the obtained values corresponding to original time series of Ref. [49] with resolution of two decimals.

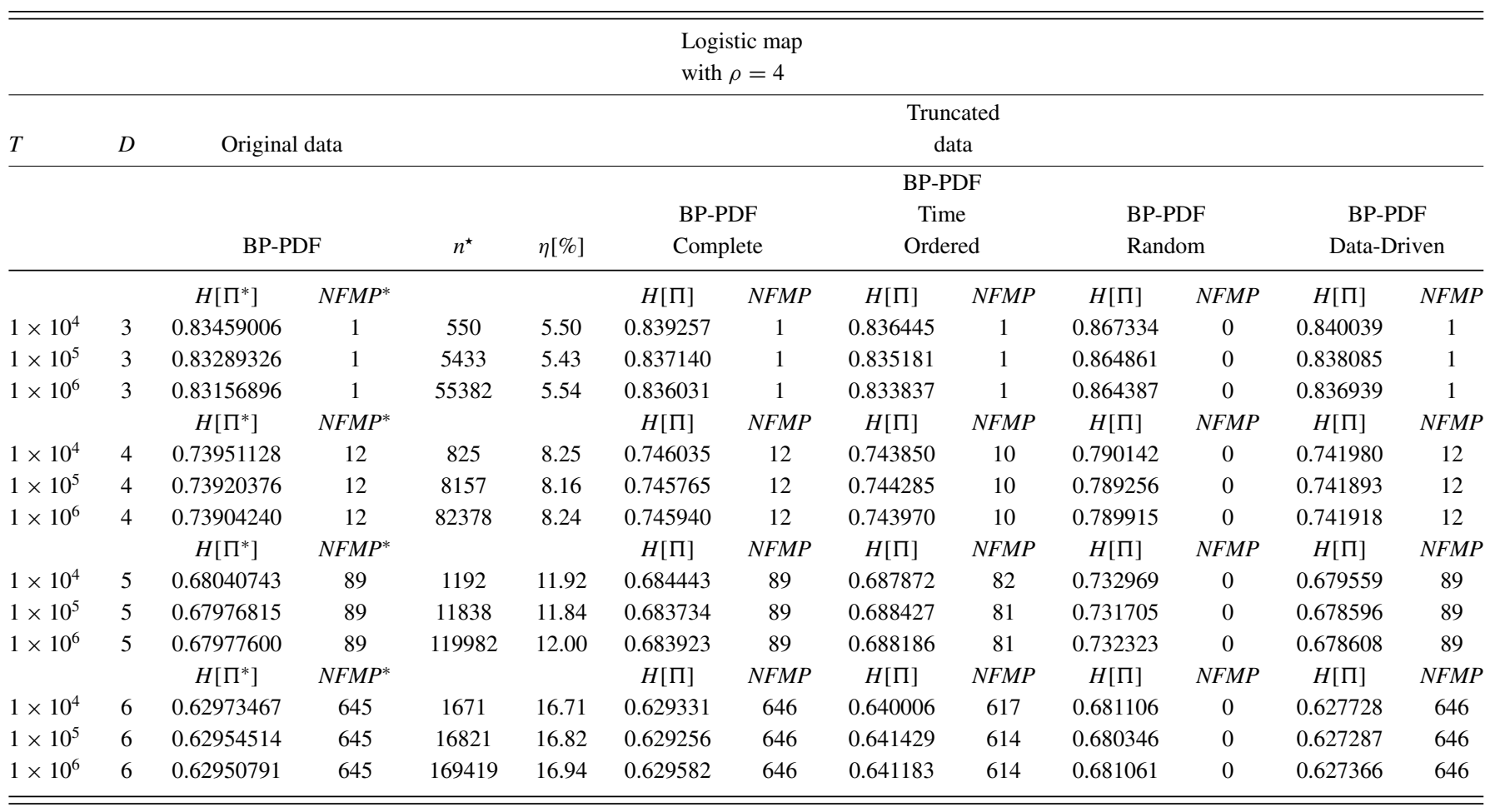


Logistic Map
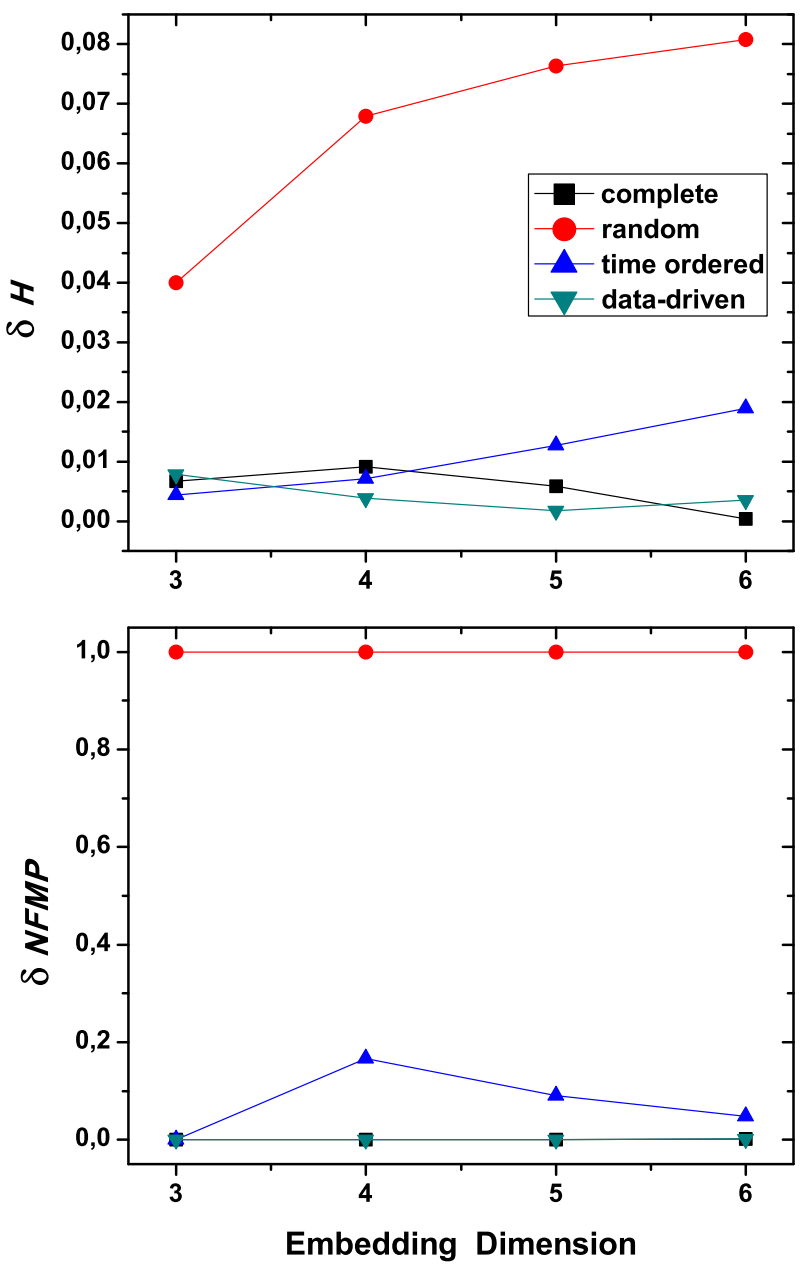

Delayed Logistic Map ( X)
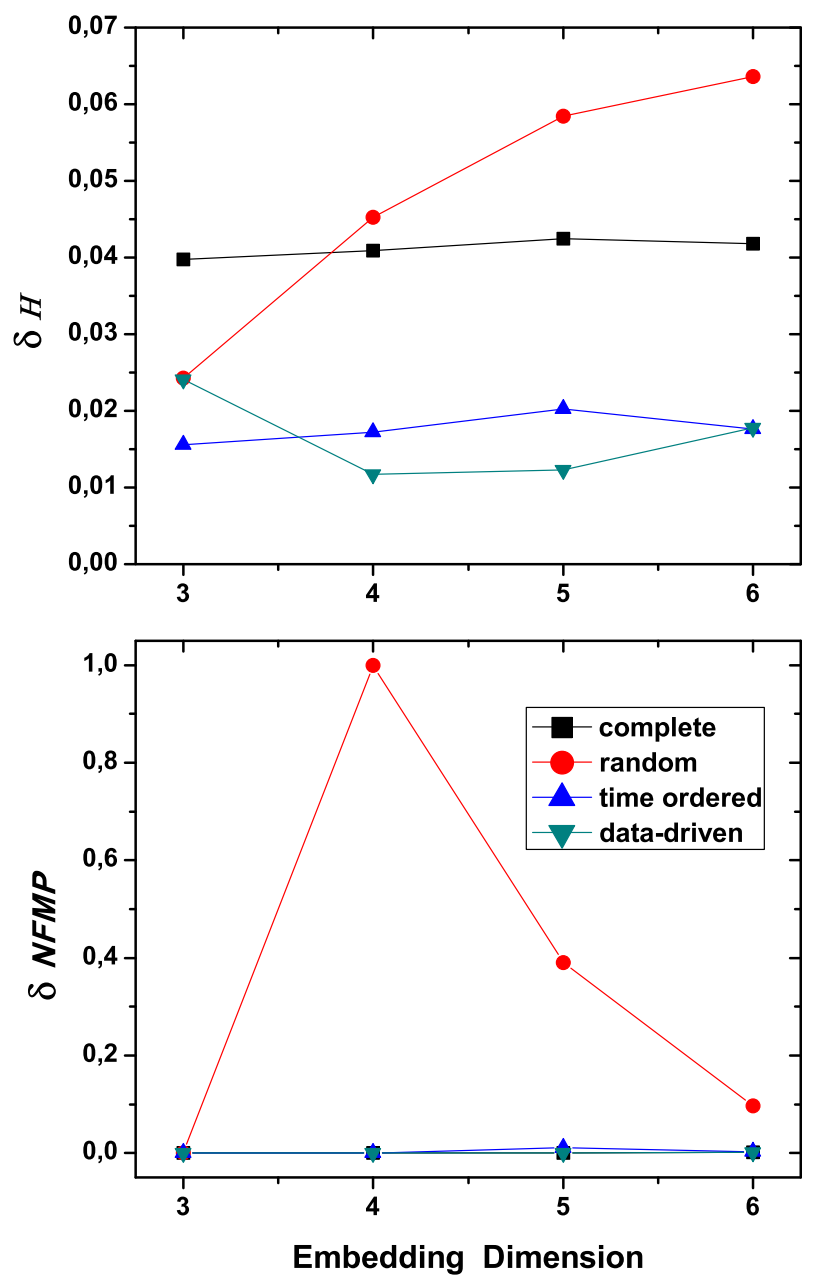

FIG. 4. Performance for the Logistic Map $\left(D_{\min }=3\right)$ and Delayed Logistic Map $(\mathrm{X})\left(D_{\min }=3\right)$.

We evaluated times series $\mathcal{X}^{*}$ of $T=10^{5}$ iteration data (after discarding $10^{4}$ iterations for stability) from the chaotic maps considered by Rosso and coworkers ${ }^{49}$ (see supplementary material). They were produced with IEEE 754 double precision floating point numbers. The corresponding values for $\Upsilon\left(\Pi^{*}\right)$ were taken from the same reference. The coarse version (low precision) time series $\mathcal{X}$ are obtained truncating the original values $x_{t}^{*}$ to two decimal digits, and then $\Upsilon\left(\Pi^{(i)}\right)$ was computed.

Figure 4 presents typical $\delta H$ and $\delta N F M P$ for the logistic and the delayed logistic maps. The supplementary material presents the results obtained for all the chaotic maps considered, grouped by their corresponding $D_{\min }$ value.

As mentioned in the Introduction, chaotic time series display forbidden ordinal patterns. However, failing to observe a given specific ordinal pattern in a time series does not necessarily imply that this pattern is forbidden; its absence qualifies the pattern just as a missing one. This could happen because the time series is not long enough or, as we see below, it was recorded with low precision.

Usually, observing or not an ordinal pattern strongly depends on the time series length $T$. Thus, if the number of non-observed ordinal patterns is constant for increasing values of the time series $T$, one can speak of a "true" forbidden (missing) ordinal patterns.

Table VII summarizes the results for the logistic map with parameter $\rho=4$, corresponding to full chaotic behavior, for different values of embedding dimension $D$, as well as the influence the time series length $T$ exerts on it. Amigó and coworkers ${ }^{50,51}$ showed the sequence of any three consecutive values satisfying $x_{i+2}^{*}<x_{i+1}^{*}<x_{i}^{*}$ never appears in this particular map. That is, the permutation pattern $\tilde{\pi}^{(D=3)}=321$ is forbidden by the system dynamics. For a fixed pattern length $D$, the number of forbidden/missing patterns (NFMP) is independent of the time series length $T$. In addition, as shown by Rosso and coworkers, ${ }^{49}$ the NFMP grows exponentially with the pattern length $D$ in the case of finite time series. Note also that the corresponding values of permutation normalized entropy, $H\left[\Pi^{*}\right]$ for a fixed value of $D$ tends to stabilize as the time series length increases.

It is expected that good reconstruction techniques will preserve, to some extent, this behavior. When we work with the truncated version of the time series, we observe that the percentage of vectors of dimension $D$ that have equal component values, $\eta$, is quite stable for different time series length $T$, with maximum value $\sim 17 \%$ for $D=6$. Comparing the 
values of $N F M P$ obtained for the truncated version with the original time series, we observe that in the case of BP-PDF Complete and BP-PDF Data-Driven are equal, except in the case of $D=6$ in which it differs in one pattern. The reason for this difference is that the pattern $\tilde{\pi}^{(D=6)}=234516$ can be found with non-zero probability in the original time series, but it is not observed in the truncated series. Note also that NFMP for the other methodologies present in general lower values than those for BP-PDF Data-Driven. As observed previously, the estimated permutation normalized entropy $H[\Pi]$ for a fixed value of $D$ tends to stabilize as the time series length increases.

One of the most successful applications of Information Theory descriptors is the task of distinguishing noise from chaos. The latter is characterized, among other features, by the presence of forbidden patterns; we denoted $D_{\min }$ the minimum embedding dimension for which there are $N F M P \neq 0$ in finitesize time series. We observed that $D_{\min }$ is preserved in all the dynamics here considered for the four reconstruction techniques.

Any reconstruction technique that introduces forbidden/missing patterns is likely to lead to the erroneous conclusion that the underlying dynamics is more random than the true one. In a complementary fashion, the Complete technique is likely to introduce more forbidden/missing patterns than the actual ones; its use may lead to infer that the system is less stochastic than it is. We thus conclude that Random Imputation and Complete should not be used. The other two techniques are competitive, but in some cases, Data-Driven outperforms Time Ordered Imputation.

Although we do not have a definitive rule for the use of the techniques presented in this work, our recommendation is that imputation rules should not be applied when $35 \%$ or more of the vectors have ties. If that is the case, any Data-Driven imputation technique may produce misleading estimates since they come only from the available evidence hiding, thus, the true underlying dynamics.

\section{SUPPLEMENTARY MATERIAL}

See supplementary material for the considered chaotic systems and their parameters, and a quantitative assessment of techniques here presented for low-precision data.

${ }^{1}$ R. H. Shumway and D. S. Stoffer, Time Series Analysis and its Applications (Springer, Berlin, 2006).

${ }^{2} \mathrm{C}$. Bandt and B. Pompe, "Permutation entropy: A natural complexity measure for time series," Phys. Rev. Lett. 88, 174102 (2002).

${ }^{3}$ O. A. Rosso, H. A. Larrondo, M. T. Martín, A. Plastino, and M. A. Fuentes, "Distinguishing noise from chaos," Phys. Rev. Lett. 99, 154102 (2007).

${ }^{4}$ Y. Cao, W. W. Tung, J. B. Gao, V. A. Protopopescu, and L. M. Hively, "Detecting dynamical changes in time series using the permutation entropy," Phys. Rev. E 70(4), 046217 (2004).

${ }^{5}$ C. Bandt, "Ordinal time series analysis," Ecol. Modell. 182, 229-238 (2005).

${ }^{6}$ A. M. Kowalski, M. T. Martín, A. Plastino, and O. A. Rosso, "BandtPompe approach to the classical-quantum transition," Phys. D 233, 21-31 (2007).

${ }^{7}$ L. Zunino, M. C. Soriano, I. Fischer, O. A. Rosso, and C. R. Mirasso, "Permutation information-theory approach to unveil delay dynamics from time-series analysis," Phys. Rev. E 82, 046212 (2010).

${ }^{8}$ O. A. Rosso, L. De Micco, A. Plastino, and H. Larrondo, "Info-quantifiers' map-characterization revisited," Phys. A 389, 249-262 (2010).
${ }^{9}$ A. M. Kowalski, M. T. Martín, A. Plastino, and O. A. Rosso, "Fisherinformation description of the classical-quantal transition," Phys. A 390, 2435-2441 (2011).

${ }^{10}$ L. De Micco, J. G. Fernández, H. A. Larrondo, A. Plastino, and O. A. Rosso, "Sampling period, statistical complexity, and chaotic attractors," Phys. A 391, 2564-2575 (2012).

${ }^{11}$ L. Zunino, M. C. Soriano, and O. A. Rosso, "Distinguishing chaotic and stochastic dynamics from time series by using a multiscale symbolic approach," Phys. Rev. E 86, 046210 (2012).

${ }^{12}$ M. C. Soriano, L. Zunino, O. A. Rosso, I. Fischer, and C. R. Mirasso, "Time scales of a chaotic semiconductor laser with optical feedback under the lens of a permutation information analysis," IEEE J. Quantum Electron. 47, 252-261 (2011).

${ }^{13}$ L. Zunino, O. A. Rosso, and M. C. Soriano, "Characterizing the hyperchaotic dynamics of a semiconductor laser subject to optical feedback via permutation entropy,” IEEE J. Sel. Top. Quantum Electron. 17(5), 1250-1257 (2011).

${ }^{14}$ J. P. Toomey and D. M. Kane, "Mapping the dynamic complexity of a semiconductor laser with optical feedback using permutation entrop," Opt. Express 22(2), 171-1725 (2014).

${ }^{15}$ L. Yang, W. Pan, L. Yan, B. Luo, and N. Li, "Mapping the dynamic complexity and synchronization in unidirectionally coupled external-cavity semiconductor lasers using permutation entropy,” J. Opt. Soc. Am. B 32(7), 1463-1470 (2015).

${ }^{16} \mathrm{H}$. Liu, B. Ren, Q. Zhao, and N. Li, "Characterizing the optical chaos in a special type of small networks of semiconductor lasers using permutation entropy,” Opt. Commun. 359, 7-84 (2016).

${ }^{17}$ H. Lange, O. A. Rosso, and M. Hauhs, "Ordinal pattern and statistical complexity analysis of daily stream flow time series," Eur. Phys. J. Special Topics 222, 535-552 (2013).

${ }^{18}$ F. Serinaldi, L. Zunino, and O. A. Rosso, "Complexity-entropy analysis of daily stream flow time series in the continental United States," Stoch. Environ. Res. Risk Assess 28, 1685-1708 (2014).

${ }^{19}$ T. Stosic, L. Telesca, D. V. De Souza Ferreira, and B. Stosic, "Investigating anthropically induced effects in streamflow dynamics by using permutation entropy and statistical complexity analysis: A case study," J. Hydrol. 540, 1136-1145 (2016).

${ }^{20}$ G. Consolini and P. De Michelis, "Permutation entropy analysis of complex magnetospheric dynamics," J. Atmos. Sol.-Terr. Phys. 115-116, 25-31 (2014).

${ }^{21}$ P. M. Saco, L. C. Carpi, A. Figliola, E. Serrano, and O. A. Rosso, "Entropy analysis of the dynamics of El $\mathrm{Ni}$ no/Southern Oscillation during the Holocene," Phys. A 389, 5022-5027 (2010).

${ }^{22}$ S. Sippel, H. Lange, M. D. Mahecha, M. Hauhs, P. Bodesheim, T. Kaminski, F. Gans, and O. A. Rosso, "Diagnosing the dynamics of observed and simulated ecosystem gross primary productivity with time causal information theory quantifiers," PLoS ONE 11(10), e0164960 (2016).

${ }^{23}$ R. Yan, Y. Liu, and R. X. Gao, "Permutation entropy: a nonlinear statistical measure for status characterization of rotary machines," Mech. Syst. Signal Process. 29, 474-484 (2012).

${ }^{24}$ A. L. L Aquino, T. S. G Cavalcante, E. S. Almeida, A. C. Frery, and O. A. Rosso, "Characterization of vehicle behavior with information theory," Eur. Phys. J. B 88, 257 (2015).

${ }^{25}$ A. L. L Aquino, H. S. Ramos, A. C. Frery, L. P. Viana, T. S. G Cavalcante, and O. A. Rosso, "Characterization of electric load with information theory quantifiers," Phys. A 465, 277-284 (2017).

${ }^{26}$ F. O. Redelico, F. Traversaro, N. Oyarzabal, I. Vilaboa, and O. A. Rosso, "Evaluation of the status of rotary machines by time causal information theory quantifiers," Phys. A 470, 321-329 (2017).

${ }^{27}$ O. A. Rosso, R. Ospina, and A. C. Frery, "Classification and verification of handwritten signatures with time causal information theory quantifiers," PLoS ONE 11(12), e0166868 (2016)..

${ }^{28}$ L. De Micco, C. M. González, H. A. Larrondo, M. T. Martín, A. Plastino, and O. A. Rosso, "Randomizing nonlinear maps via symbolic dynamics," Phys. A 387, 3373-3383 (2008).

${ }^{29}$ L. De Micco, H. A. Larrondo, A. Plastino, and O. A. Rosso, "Quantifiers for randomness of chaotic pseudo-random number generators," Philos. Trans. R. Soc. A 367, 3281-3296 (2009).

${ }^{30}$ M. Zanin, L. Zunino, O. A. Rosso, and D. Papo, "Permutation entropy and its main biomedical and econophysics applications: A review," Entropy 14, 1553-1577 (2012).

${ }^{31} \mathrm{X}$. Li, G. Ouyang, and D. A. Richards, "Predictability analysis of absence seizures with permutation entropy," Epilepsy Res. 77(1), 70-74 (2007). 
${ }^{32}$ X. Li, S. Cui, and L. J. Voss, "Using permutation entropy to measure the electroencephalographic effects of sevoflurane," Anesthesiology 109, 448-456 (2008).

${ }^{33}$ U. Parlitz, S. Berg, S. Luther, A. Schirdewan, J. Kurths, and N. Wessel, "Classifying cardiac biosignals using ordinal pattern statistics and symbolic dynamics," Comput. Biol. Med. 42, 319-327 (2012).

${ }^{34}$ F. C. Morabito, D. Labate, F. La Foresta, A. Bramanti, G. Morabito, and I. Palamara, "Multivariate multi-scale permutation entropy for complexity analysis of Alzheimer's disease EEG,” Entropy 14, 1186-1202 (2012).

${ }^{35}$ J. Li, J. Yan, X. Liu, G. Ouyang, "Using permutation entropy to measure the changes in EEG signals during absence seizures," Entropy 16, 3049-3061 (2014).

${ }^{36}$ F. Montani, E. B. Deleglise, and O. A. Rosso, "Efficiency characterization of a large neuronal network: A causal information approach," Phys. A 401, 58-70 (2014).

${ }^{37}$ F. Montani and O. A. Rosso, "Entropy-complexity characterization of brain development in chickens," Entropy 16, 4677-4692 (2014).

${ }^{38}$ Z. Liang, Y. Wang, X. Sun, D. Li, L. J. Voss, J. W. Sleigh, S. Hagihira, and X. Li, "EEG entropy measures in anesthesia," Front. Comput. Neurosci. 9, 16 (2015).

${ }^{39}$ F. Montani, R. Baravalle, L. Montangie, and O. A. Rosso, "Causal information quantification of prominent dynamical features of biological neurons," Philos. Trans. R. Soc. A 373(2056), 20150109 (2015).

${ }^{40}$ F. Montani, O. A. Rosso, F. S. Matias, S. L. Bressler, and C. R. Mirasso, "A symbolic in-formation approach to determine anticipated and delayed synchronization in neuronal circuit models," Philos. Trans. R. Soc.A 373(2056), 20150110 (2015).

${ }^{41}$ L. Zunino, M. Zanin, B. M. Tabak, D. G. Pérez, and O. A. Rosso, "Forbidden patterns, permutation entropy and stock market inefficiency," Phys. A 388(14), 2854-2864 (2009).

${ }^{42}$ L. Zunino, M. Zanin, B. M. Tabak, D. G. Pérez, and O. A. Rosso, "Complexity-entropy causality plane: A useful approach to quantify the stock market inefficiency," Phys. A 389(9), 1891-1901 (2010).

${ }^{43}$ A. F. Bariviera, M. B. Guercio, L. B. Martinez, and O. A. Rosso, "The (in)visible hand in the Libor market: An information theory approach," Eur. Phys. J. B 88, 208 (2015).

${ }^{44}$ A. F. Bariviera, M. B. Guercio, L. B. Martinez, and O. A. Rosso, “A permutation information theory tour through different interest rate maturities: The Libor case," Philos. Trans. R. Soc. A 373(2056), 20150119 (2015).

${ }^{45}$ A. F. Bariviera, M. B. Guercio, L. B. Martinez, and O. A. Rosso, "Libor at crossroads: Stochastic switching detection using information theory quantifiers," Chaos Solitons Fractals 88, 172-182 (2016).

${ }^{46}$ L. Zunino, A. F. Bariviera, M. B. Guercio, L. B. Martinez, and O. A. Rosso, "Monitoring the informational efficiency of European corporate bond markets with dynamical permutation min-entropy," Phys. A 456, 1-9 (2016).

${ }^{47}$ O. A. Rosso, L. C. Carpi, P. M. Saco, M. Gómez Ravetti, A. Plastino, and H. Larrondo, "Causality and the entropy-complexity plane: Robustness and missing ordinal patterns," Phys. A 391, 42-55 (2012).
${ }^{48}$ O. A. Rosso, L. C. Carpi, P. M. Saco, M. Gómez Ravetti, H. Larrondo, and A. Plastino, "The Amigó paradigm of forbidden/missing patterns: A detailed analysis,” Eur. Phys. J. B 85, 419-430 (2012).

${ }^{49}$ O. A. Rosso, F. Olivares, L. Zunino, L. De Micco, A. L. L Aquino, A. Plastino, and H. A. Larrondo, "Characterization of chaotic maps using the permutation Bandt-Pompe probability distribution," Eur. Phys. J. B 86, 116-129 (2013).

${ }^{50}$ J. M. Amigó, S. Zambrano, and M. A. F Sanjúan, "True and false forbidden patterns in deterministic and random dynamics," Europhys. Lett. 79, 50001 (2007).

${ }^{51}$ J. M. Amigó, Permutation Complexity in Dynamical Systems (Springer, Berlin, 2010)

${ }^{52}$ L. C. Carpi, P. M. Saco, and O. A. Rosso, "Missing ordinal patterns in correlated noises," Phys. A 389, 2020-2029 (2010).

${ }^{53} \mathrm{H}$. Wold, A Study in the Analysis of Stationary Time Series (Almqvist and Wiksell, Upsala, 1938).

${ }^{54}$ S. Cambanis, C. D. Hardin, and A. Weron, "Innovations and Wold decompositions of stable sequences," Probab. Theory Relat. Fields 79, 1-27 (1988).

${ }^{55}$ L. Zunino, F. Olivares, F. Scholkmann, and O. A. Rosso, "Permutation entropy based time series analysis: Equalities in the input signal can lead to false conclusions," Phys. Lett. A 381, 1883-1892 (2017).

${ }^{56}$ C. Bian, C. Qin, Q. Y. D Ma, and Q. Shen, "Modified permutation-entropy analysis of heartbeat dynamics," Phys. Rev. E 85, 021906 (2012).

${ }^{57}$ M. Riedl, A. Müller, and N. Wessel, "Practical considerations of permutation entropy," Eur. Phys. J. Special Topics 222, 249-262 (2013).

${ }^{58} \mathrm{C}$. Bandt and F. Shiha, "Order patterns in time series," J. Time Ser. Anal. 28, 646 (2007)

${ }^{59}$ F. Olivares, A. Plastino, and O. A. Rosso, “Ambiguities in Bandt-Pompe's methodology for local entropic quantifiers," Phys. A 391, 2518-2526 (2012).

${ }^{60}$ O. A. Rosso, F. Olivares, and A. Plastino, "Noise vs. chaos in a causal Fisher-Shannon plane," Pap. Phys. 7, 070006 (2015).

${ }^{61}$ J. B. Brissaud, "The meaning of entropy," Entropy 7, 68-96 (2005).

${ }^{62}$ C. E. Shannon, "A mathematical theory of communication," Bell Syst. Technol. J. 27, 379-423 (1948). 623-656.

${ }^{63}$ C. E. Shannon and W. Weaver, The Mathematical Theory of Communication (University of Illinois Press, Champaign, IL, 1949).

${ }^{64}$ A. R. Donders, G. J. van der Heijden, T. Stijnen, and K. G. Moons, "Review: A gentle introduction to imputation of missing values," J. Clin. Epidemiol. 59, 1087-1091 (2006).

${ }^{65} \mathrm{C}$. Masoller and O. A. Rosso, "Quantifying the complexity of the delayed logistic map,” Philos. Trans. R. Soc. A 369, 425-438 (2011).

${ }^{66}$ B. Luque, L. Lacasa, F. Ballesteros, and J. Luque, "Horizontal visibility graphs: Exact results for random time series," Phys. Rev. E 80, 046103 (2009). 